\title{
АЛГОРИТМ КОНСТРУИРОВАНИЯ ИСКУССТВЕННЫХ ЗУБНЫХ РЯДОВ ПРИ ПРОГЕНИЧЕСКОМ И ПРОГНАТИЧЕСКОМ СООТНОШЕНИИ ФРОНТАЛЬНЫХ СЕГМЕНТОВ БЕЗЗУБЫХ ЧЕЛЮСТЕЙ
}

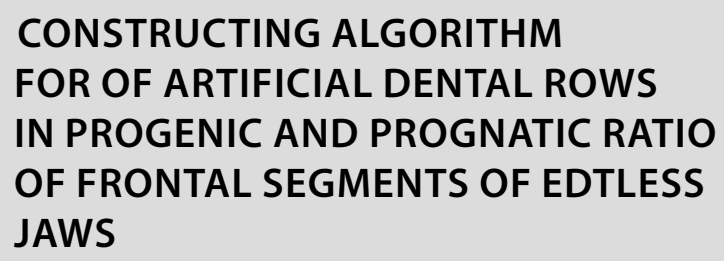

CONSTRUCTING ALGORITHM FOR OF ARTIFICIAL DENTAL ROWS IN PROGENIC AND PROGNATIC RATIO OF FRONTAL SEGMENTS OF EDTLESS JAWS

D. Aksyonov

Yu. Mayboroda

I. Aksenov

Summary. The purpose of this study is to develop, on the basis of mathematical calculations, the optimal options for designing artificial dentition in the frontal segments of the edentulous jaws with a progenic and prognathic ratio. For mathematical calculations, the digital parameters of 260 pairs of plaster models of the jaws were used, on the basis of which the average arch for the upper and lower jaws and the angles of the alveolar ridges of the frontal segments of the jaws were calculated. On the basis of anthropometric segmental digital data on the sizes of the alveolar arches of the plaster models of the edentulous upper and lower jaws, a mathematical model has been developed that allows in each case to design artificial dentition with a prognathic and progenic ratio of the frontal segments of the jaws, taking into account the level of the degree of atrophic changes in the tops of the alveolar ridges and angles slope of the tops of the interalveolar ridges.

The obtained digital data of calculations for various variants of the ratio of the alveolar processes makes it possible to optimally determine the variants of the middle interalveolar lines in the frontal segments of the edentulous jaws for a more rational placement of artificial teeth.

Keywords: mathematical model, progeny, prognathia, edentulous jaws, alveolar processes.

\author{
Аксёнов Даниил Игоревич \\ Аспирант, Ставропольский Государственный \\ Медицинский Университет \\ p.ghost@rambler.ru \\ Майборода Юрий Николаевич \\ Дочент, Ставропольский Государственный \\ Медицинский Университет \\ Аксёнов Игорь Николаевич \\ Ассистент, Ставропольский Государственный \\ Медицинский Университет
}

Аннотация. Целью данного исследования является разработка на основе математических расчётов, оптимальные варианты конструирования искусственных зубных рядов во фронтальных сегментах беззубых челюстей при прогеническом и прогнатическом соотношении. Для математических расчётов были использованы цифровые параметры 260 пар гипсовых моделей челюстей, на основе которых рассчитывались средние дуги для верхней и нижней челюстей и величина углов альвеолярных гребней фронтальных сегментов челюстей. На основе антропометрических сегментарных цифровых данных размеров альвеолярных дуг гипсовых моделей беззубых верхних и нижних челюстей, разработанная математическая модель, которая позволяет в каждом конкретном случае конструировать искусственные зубные ряды при прогнатическом и прогеническом соотношении фронтальных сегментов челюстей с учётом уровня степени атрофических изменений вершин альвеолярных гребней и углов наклона вершин межальвеолярных гребней.

Полученные цифровые данные расчётов при различных вариантах соотношения альвеолярных отростков позволяет оптимально определить варианты средних межальвеолярных линий во фронтальных сегментах беззубых челюстей для более рациональной расстановки искусственных зубов.

Ключевые слова: математическая модель, прогения, прогнатия, беззубые челюсти, альвеолярные отростки.

ложа происходят изменения альвеолярных отростков с различной степенью атрофии и уровня податливости слизистой оболочки, которые необходимо учитывать при планировании конструкции полных съёмных протезов. Подобный подход означает необходимость практического осуществления принципа индивидуального протезирования, при котором конструирование и изготовление зубных протезов являются заключительными этапами ортопедического лечения при полной потере зубов. [6,7,13] 


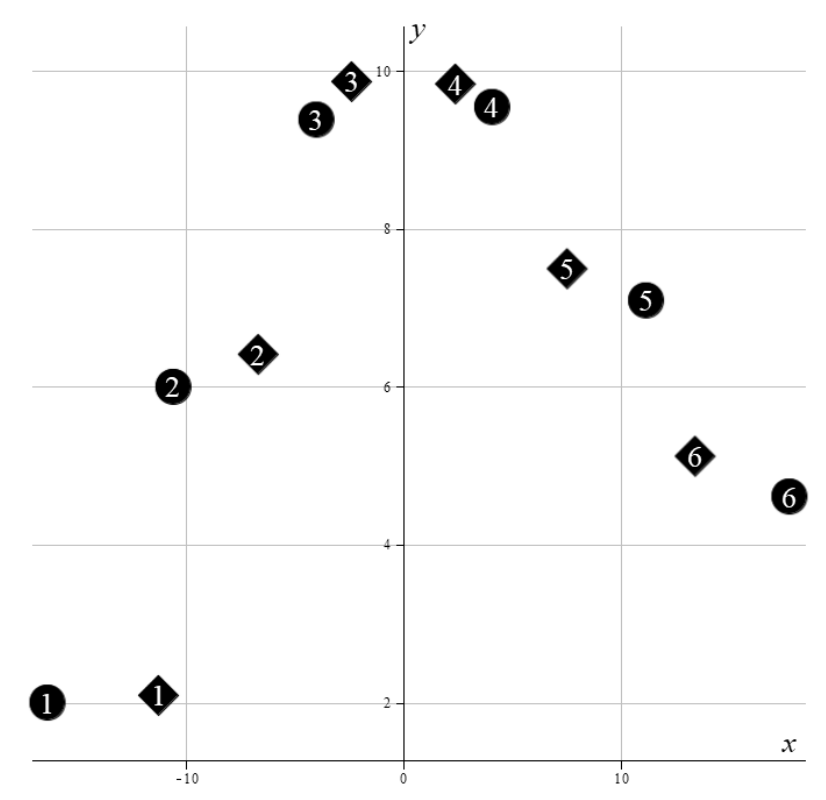

Круглыми точками обозначены зубы верхней челюсти, ромбами обозначены зубы нижней челюсти: 1 - левый клык, 2 - левый боковой резец, 3 - левый центральный резец, 4 - правый центральный резец, 5 - правый боковой резец, 6 - правый клык

Рис. 1. Выбор системы координат.

\section{^итературный обзор}

Протезы, изготовленные при недостаточном учёте морфологических особенностей тканей протезного ложа, способствуют усилению атрофических процессов альвеолярных гребней особенно на фоне малоподатливой слизистой оболочки $[1,8,14,16,19]$. Особенно неблагоприятными клиническими условиями протезирования отмечаются при прогнатическом и прогеническом соотношении альвеолярных отростков фронтальных сегментов челюстей $[12,17]$ в сочетании вариантов диспропорций альвеолярных отростков жевательных и фронтальных сегментов челюстей $[9,18]$. Последнее затрудняет выбор конструирования искусственных зубных рядов, который во многом зависит от правильной разметки гипсовых моделей беззубых челюстей для восстановления контуров зубной дуги, имеющимся до потери зубов и атрофии альвеолярных отростков [4]. Путём измерений альвеолярных отростков моделей челюстей с зубами были установлены некоторые анатомо-топографические закономерности, которые в некоторой степени возможны при конструировании искусственных зубов в ортогнатическом прикусе.[11]

Способы конструирования зубных рядов в полных съёмных протезах при прогеническом соотношении беззубых челюстей основаны на эмпирических ориентирах размеров челюстей [15] и обусловлены отсут- ствием научно-обоснованных рекомендаций, позволяющих с математических позиций оптимизировать конструкцию зубных рядов при различных уровнях соотношения во фронтальных сегментах челюстей и выработать алгоритм планирования ортопедического лечения больных. Существующие до настоящего времени методы конструирования искусственных зубных рядов несут в себе элементы больших погрешностей и, тем более, вопрос протезирования пациентов при прогеническом или прогнатическом соотношении беззубых челюстей на фоне аномалий развития, а также атрофических процессов до сих пор остаются открытыми. Также с позиции количественных характеристик [20] имеющиеся литературные данные полностью не освещают проблему вопросов симметрии или асимметрии и остаются актуальными для практической стоматологии. Поэтому определение углов расхождения во фронтальных участках на основе данных вариантов пересечения межальвеолярных линий методом математических расчётов является актуальный, т.к. позволяет разработать оптимальную индивидуальную расстановку зубов. Целесообразность данных исследований необходима в связи с наличием определённых трудностей в оценке морфологических характеристик тканевых компонентов вершин альвеолярных гребней из-за их неравномерной резорбции. Поэтому совершенствование конструирования зубных рядов в настоящее время невозможна без антропологического подхода. [2] 


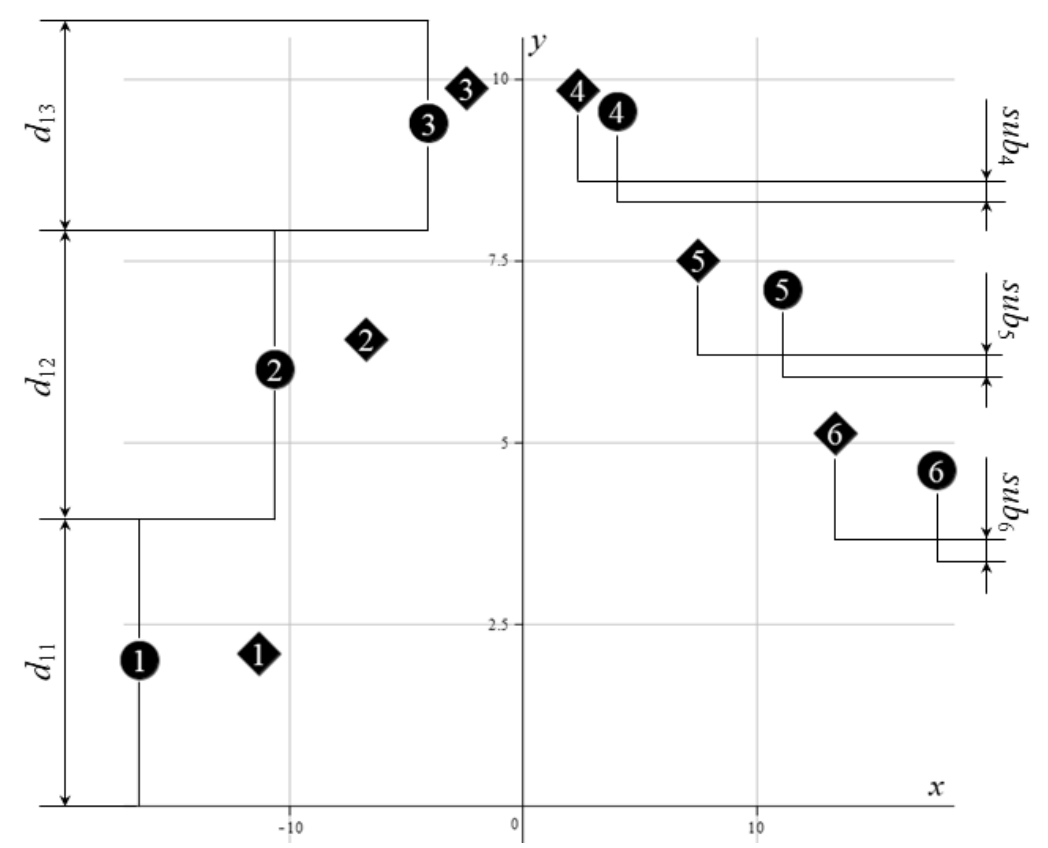

Рис. 2. Исходные данные

\section{Материалы и метолы}

Антропометрические измерения 260 пар диагностических гипсовых моделей беззубых пациентов послужили основой для разработки математической модели при различных вариантах соотношения альвеолярных дуг фронтальных сегментов челюстей. Создана программа для оптимизации конструирования искусственных зубных рядов при прогеническом и прогнатическом вариантах их топографического соотношения.

\section{Результаты}

В качестве основных математических задач рассматривают систему построения межальвеолярной средней линии между дугами фронтальных сегментов верхней и нижней челюсти с вычислением углов между центральными резцами, боковыми верхними и нижними резцами, и верхними и нижними клыками. Для решения данной проблемы в первую очередь выбирают систему координат следующим образом: ось абсцисс $(O x)$ проходит через начало левого клыка верхней челюсти, ось $(O y)$ проходит через стык центральных резцов верхней челюсти. Для удобства зубы верхней и нижней челюстей нумеруют следующим образом: левому клыку присваивают номер 1, левому боковому резцу - номер 2, левому центральному резцу - номер 3. Продолжают нумерацию для правой стороны в обратном порядке: правому центральному резцу номер 4, правому боковому резцу - номер 5, правому клыку номер 6 (рис. 1).
Для того чтобы построить дуги нижней и верхней челюстей необходимо в точности определить положение зубов. В данной математической модели под зубами понимают их геометрические центры, что приведет к рассмотрению зубов как точек на координатной плоскости. Исходными данными, описываемого далее алгоритма построения средней дуги, являются: для верхней челюсти - вертикальные расстояния между горизонтальными линиями, проходящими через начала зубов верхней челюсти: $d_{11}$ - вертикальное расстояние, соответствующее левому клыку, $d_{12}-$ вертикальное расстояние, соответствующее левому боковому резцу, $d_{13}$ - вертикальное расстояние, соответствующее левому центральному резцу, $d_{14}$ - вертикальное расстояние, соответствующее правому центральному резцу, $d_{15}$ - вертикальное расстояние, соответствующее правому боковому резцу и $d_{16}$ - вертикальное расстояние, соответствующее правому клыку. Двойная индексация используется для отделения соответствующих величин верхней и нижней челюстей: если первый индекс равен «1», то подразумевается верхняя челюсть, если «2»- нижняя. Для нижней челюсти исходными данными являются вертикальные расстояния между верхними и нижними альвеолярными гребнями, т.е. расстояния между горизонтальными линиями, проходящими через начала соответствующих верхних и нижних зубов: $s u b_{1}$ - расстояние между левыми клыками, $s u b_{2}$ - расстояние между левыми боковыми резцами, $s u b_{3}$ - расстояние между левыми центральными резами, $s u b_{4}$ - расстояние между правыми центральными резцами, $s u b_{5}$ - расстояние между правыми боковыми 
Таблица 1. Сводные данные средних размеров коронок постоянных зубов, мм по В.Д. Устименко

\begin{tabular}{|l|l|l|l|}
\hline Название зуба & Номер зуба, $i$ & Ширина $c_{i,}$ мм & Высота $h_{i}$ мм \\
\hline Верхний клык & 1,6 & 7,6 & 8,9 \\
\hline Верхний боковой резец & 2,5 & 6,5 & 7,8 \\
\hline Верхний центральный резец & 3,4 & 8,5 & 8,9 \\
\hline Нижний клык & 1,6 & 6,7 & 9,4 \\
\hline Нижний боковой резец & 2,5 & 6,0 & 7,9 \\
\hline Нижний центральный резец & 3,4 & 5,3 & 7,8 \\
\hline
\end{tabular}

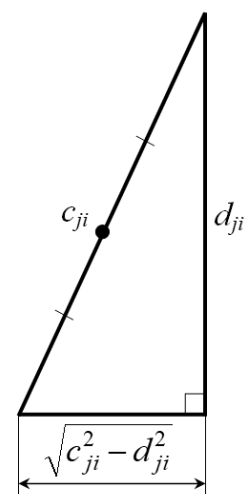

Рис. 3. Вычисление горизонтальной составляющей зуба

резцами и $s u b_{6}$ - расстояние между правыми клыками (рис. 2). Как уже отмечалось ранее, круглыми точками обозначены зубы верхней челюсти, ромбами - нижней в декартовой системе координат.

В каждом конкретном случае указанные расстояния задают дуги, соответствующие верхнему и нижнему альвеолярным гребням. Однако для точного определения положения зубов на координатной плоскости необходимо знать размеры коронок постоянных зубов, представленные в таблице 1.

При решении поставленной задачи определяют координаты зубов в выбранной декартовой системе. (рис. 2). Исходные данные позволяют в первую очередь определить координаты $x_{1 i}$ и $y_{l i} i=1.6$, каждого из зубов верхней челюсти. Для определения ординат зубов верхней челюсти потребуется дополнительная величина $P e a k_{u p}$ определяющая насколько далеко выступает верхняя челюсть относительно оси $O x$ :

$$
\text { Peak }_{u p}=d_{11}+d_{12}+d_{13}
$$

Тогда в соответствии с введенными обозначениями ординаты $y_{1 i}$ зубов верхней челюсти могут быть вычислены по следующим формулам

$$
y_{11}=\frac{d_{11}}{2}, y_{12}=d_{11}+\frac{d_{12}}{2}, y_{13}=d_{11}+d_{12}+\frac{d_{13}}{2},
$$

$$
\begin{aligned}
& y_{14}=\text { Peak }_{u p}-\frac{d_{14}}{2}, y_{15}=\text { Peak }_{u p}-d_{14}-\frac{d_{15}}{2}, \\
& y_{16}=\text { Peak }_{u p}-d_{14}-d_{15}-\frac{d_{16}}{2} .
\end{aligned}
$$

Вычисление абсцисс $x_{1 i}$ зубов верхней челюсти проводят на основе теоремы Пифагора [10] из прямоугольного треугольника, образованного прямой, соединяющей начало зуба с его концом (на рис. 3 - гипотенуза), прямой, проходящей через начало зуба параллельно оси абсцисс $O x$ (на рис. 3 - нижний катет), и перпендикуляром, опущенным из конца зуба на прямую, проходящую через начало нижнего зуба параллельно оси абсцисс $O x$ (на рис. 3 - боковой катет).

Используя горизонтальные составляющие зубов верхней челюсти, определяют абсциссы $x_{1 i}$ зубов по следующим формулам:

$$
\begin{aligned}
& x_{13}=-\frac{1}{2} \sqrt{c_{13}^{2}-d_{13}^{2}}, x_{12}=2 x_{13}-\frac{1}{2} \sqrt{c_{12}^{2}-d_{12}^{2}}, \\
& x_{11}=x_{12}-\frac{1}{2} \sqrt{c_{12}^{2}-d_{12}^{2}}-\frac{1}{2} \sqrt{c_{11}^{2}-d_{11}^{2}}, \\
& x_{14}=\frac{1}{2} \sqrt{c_{14}^{2}-d_{14}^{2}}, x_{15}=2 x_{14}+\frac{1}{2} \sqrt{c_{15}^{2}-d_{15}^{2}}, \\
& x_{16}=x_{15}+\frac{1}{2} \sqrt{c_{15}^{2}-d_{15}^{2}}+\frac{1}{2} \sqrt{c_{16}^{2}-d_{16}^{2}} .
\end{aligned}
$$

где $c_{i}$ - ширина $i$-го зуба верхней челюсти (табл. 1). 


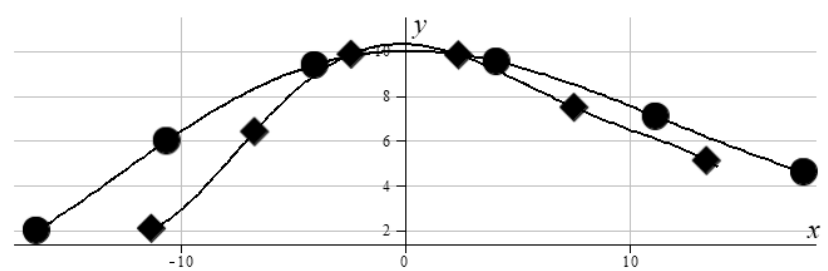

Круглыми точками обозначены зубы верхней челюсти, ромбами - нижней: изображение в масштабе 1:1

Рис. 4. Получение дуг верхней и нижней челюсти.

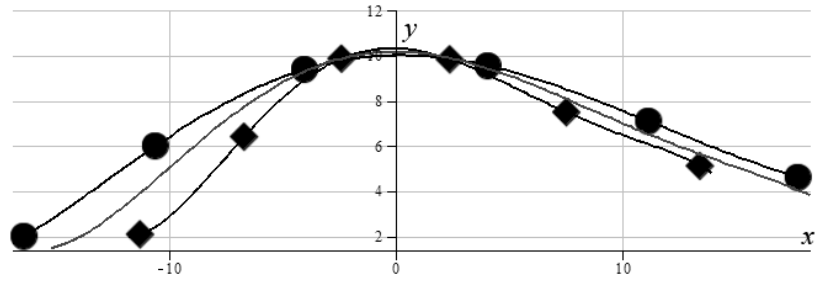

Круглыми точками обозначены зубы верхней челюсти, ромбами - нижней, Изображение в масштабе $1: 1$

Рис. 5. Получение средней линии для дуг верхней и нижней челюстей

По такому же принципу вычисляют абсциссы для зубов фронтального сегмента нижней челюсти.

На основе исходных данных координат и абсцисс строят кривые альвеолярных дуг по интерполяционной формуле Лагранжа [5]

Интерполяционная формула Лагранжа позволяет по заданным координатам точек $\left(x_{i}, y_{i}\right)$ построить кривую следующим образом:

$$
L(x)=\sum_{i=0}^{n} y_{i} \cdot l_{i}(x) \text {, где } l_{i}(x)=\prod_{i=0, j \neq i}^{n} \frac{x-x_{j}}{x_{i}-x_{j}} .
$$

В качестве исходных данных для интерполяционной формулы берутся $n$ точек, $i=1 . n$, связанных зависимостью $L(x)$.

По координатам, полученным для зубов верхней и нижней челюстей, $\left(x_{1 i}, y_{1 i}\right)$ и $\left(x_{2 i}, y_{2 i}\right)$ соответственно, $i=$ 1.6, которые и будут исходными денными, получают две кривые, которые будут соответствовать дугам верхней и нижней челюстей:

$$
\begin{aligned}
& F_{\text {up }}(x)=\sum_{i=0}^{n} y_{1 i} \cdot l_{i}(x), \text { где } l_{i}(x)=\prod_{i=0, j \neq i}^{n} \frac{x-x_{1 j}}{x_{1 i}-x_{1 j}} ; \\
& F_{\text {down }}(x)=\sum_{i=0}^{n} y_{2 i} \cdot l_{i}(x), \text { где } l_{i}(x)=\prod_{i=0, j \neq i}^{n} \frac{x-x_{2 j}}{x_{2 i}-x_{2 j}} .
\end{aligned}
$$

Результатом интерполирования будут кривые, приближающие дуги верхней и нижней челюстей. (рис. 4).
Исходные данные для построения средней линии $\left(\bar{x}_{i}, \bar{y}_{i}\right), i=1.6$, могут быть получены из соотношений:

$$
\bar{x}_{i}=\left(x_{1 i}+x_{2 i}\right) / 2, \bar{y}_{i}=\left(y_{1 i}+y_{2 i}\right) / 2,
$$

где $\left(x_{1 i}, y_{1 i}\right)$ - координаты верхних зубов, $\left(x_{2 i}, y_{2 i}\right)-$ координаты нижних зубов, $i=1.6$. Получив необходимые координаты, строят среднюю дугу (рис. 5) по формуле:

$$
F_{\text {mid }}(x)=\sum_{i=0}^{n} \bar{y}_{i} \cdot l_{i}(x) \text {, где } l_{i}(x)=\prod_{i=0, j \neq i}^{n} \frac{x-\bar{x}_{j}}{\bar{x}_{i}-\bar{x}_{j}},
$$

При построении средней линии используются те же методы, что и при построении дуг верхней и нижней челюстей.

Для расчета величины углов между рассматриваемыми зубами используют трехмерную систему координат $O x y z$ стандартным образом, добавив к введенной ранее системе координат ось $O z$, перпендикулярную линию одновременно к оси $O x$ и оси $O y$ (рис. 6а).

Для удобства расчета углов (рис. 6б) осуществляют параллельный перенос вычисленных координат на величину shift $=-\min \left(y_{11}, y_{16}, y_{21}, y_{26}\right)$ вдоль оси $O y$ :

$$
y_{j i}^{\prime}=y_{j i}+s h i f t, j=1.2, i=1.6 .
$$

Нумерация зубов остается прежней, при этом координаты $z_{2 i} i=1.6$, по добавленной оси $O z$ для зубов 


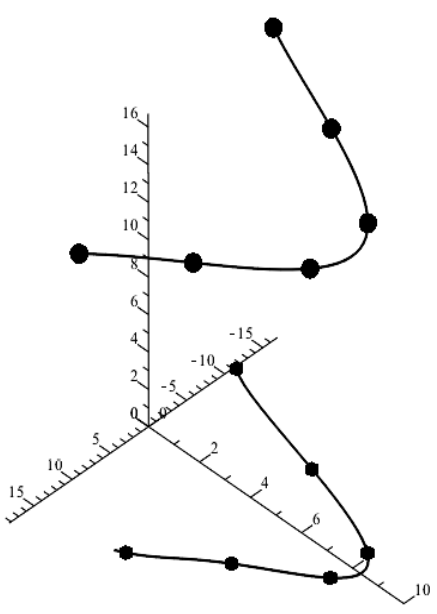

a)

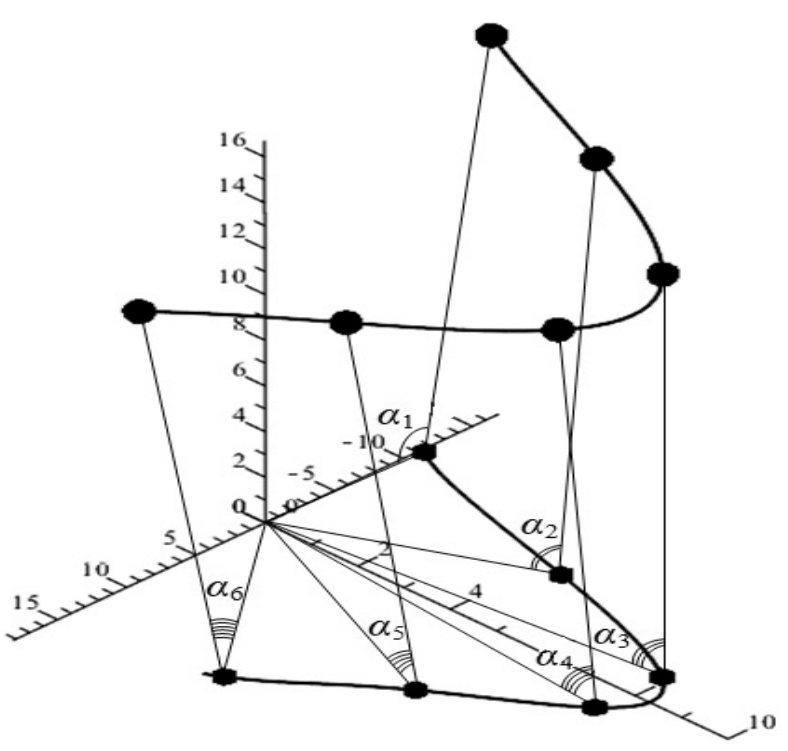

б)

Рис. 6. Получение углов: $\alpha_{1}$ - угол между левыми клыками, $\alpha_{2}-$ угол между левыми боковыми резцами, $\alpha_{3}$ - угол между левыми центральными резцами, $\alpha_{4}$-угол между правыми центральными резцами, $\alpha_{5}$ - угол между правыми боковыми резцами, $\alpha_{6}$ - угол между правыми клыками: а) трехмерная система координат; б) углы между зубами

$$
\alpha_{i}=\left\{\begin{array}{c}
\pi-\operatorname{arctg}\left(\sqrt{\frac{\left(x_{1 i}-x_{(1) 2 i}\right)^{2}+\left(y_{1 i}-y_{(1) 2 i}\right)^{2}+z_{1 i}{ }^{2}}{\left(x_{2 i}-x_{(1) 2 i}\right)^{2}+\left(y_{2 i}-y_{(1) 2 i}\right)^{2}}}\right), \text { если } x_{(1) 2 i}^{2}+y_{(1) 2 i}^{2}>x_{2 i}^{2}+y_{2 i}^{2}, \\
\operatorname{arctg}\left(\sqrt{\frac{\left(x_{1 i}-x_{(1) 2 i}\right)^{2}+\left(y_{1 i}-y_{(1) 2 i}\right)^{2}+z_{1 i}^{2}}{\left(x_{2 i}-x_{(1) 2 i}\right)^{2}+\left(y_{2 i}-y_{(1) 2 i}\right)^{2}}}\right), \text { иначе }, \\
\text { где } i=1.6 .
\end{array}\right.
$$

Рис. 8

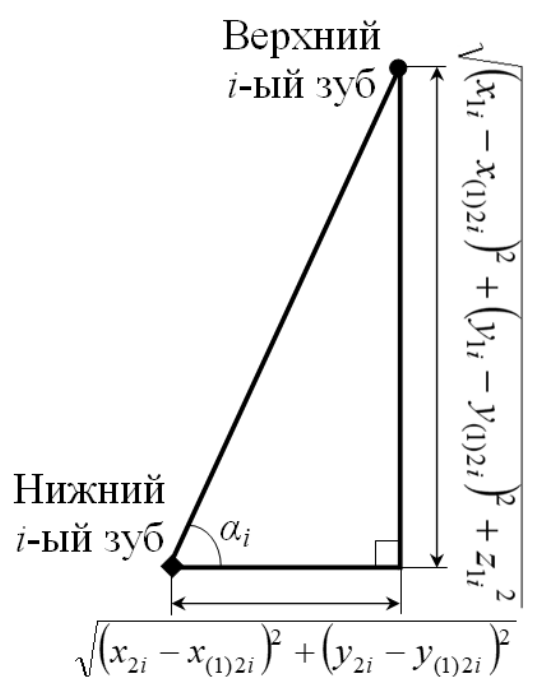

Рис. 7. Вычисление угла 
нижней челюсти будут равны 0, а для расчета координат $z_{l i}, i=1.6$, для зубов верхней челюсти, потребуется установить точку соприкосновения зубов верхней и нижней челюсти и данные о средней высоте коронки соприкасающихся зубов (табл. 1):

$$
\left\{\begin{array}{l}
z_{1 i}=h_{1 m}+h_{2 n}, \\
z_{2 i}=0,
\end{array}\right.
$$

Где тип-номера соприкасающихся верхних и нижних зубов соответственно.

Для каждой пары верхнего и нижнего соответствующих зубов величину угла рассчитывают из прямоугольного треугольника (рис. 7), образованного прямой, соединяющей верхний и нижний зубы (на рис. 7 - гипотенуза), прямой $\operatorname{Str}_{i}$ (на рис. 7 - нижний катет), и прямой, соединяющей верхний зуб с его проекцией на $\mathrm{Str}_{i}$ (на рис. 7 - боковой катет):

Искомыми углами буду углы $\alpha_{i}$ образованные линиями, соединяющими соответствующие верхние и нижние зубы и линиями, соединяющими нижние зубы с началом координат. По определению тангенса [3], находят углы для каждой пары соответствующих верхнего и нижнего зубов $\alpha_{i}$ с учетом вида угла (рис. 8).
Отметим, что для правой и левой сторон углы наклона зубов будут отличаться.

\section{Зак^Ючение}

Изучение особенностей строения альвеолярных отростков беззубых челюстей выявило разную степень изгибов и уровень угловых наклонов вершин гребневых участков фронтальных сегментов челюстей и формы межальвеолярных зубных дуг характеризуются значительной вариабельностью. На основе цифровых данных расхождения фронтальных сегментов альвеолярных отростков между обеими челюстями получены семь основных вариантов прогенического, пять прогнатического соотношения челюстей и их сочетаний.

Приведённые общие принципы математических расчётов при различных вариантах соотношения альвеолярных отростков позволяют определить варианты срединных межальвеолярных линий и, тем самым, более рационально конструировать зубные ряды во фронтальном сегменте беззубых челюстей. Детальные принципы математических расчётов для каждого из вариантов с целью оптимальной расстановки зубов будут отражены в последующих публикациях.

\section{ЛИТЕРАТУРА}

1. Авдеев Е.Н., Смирнов Е.В., Лесных Н.И., Калмыков В.В., Лихушина Т.С. Протезирование полными съемными пластиночными протезами при высоких степенях атрофии альвеолярного отростка верхней и альвеолярной части нижней челюстей. // Научные ведомости Белгородского государственного университета. Серия: Медицина. Фармация. 2014. № 24 (195). С. 265-272.

2. Божук Т.Н., Севостьянова М.А., Павлова Т.В. Распространенность форм физиологического прикуса у людей с разными типами лица. // Фундаментальные исследования. 2011. № 2. С. 44-50.

3. Гельфанд И.М., Львовский С.М., Тоом А.Л. Тригонометрия. И.М. Гельфанд, С.М. Львовский, А.Л. Тоом. Москва, 2010. (Изд. 4-е)

4. Гришечкин С.Д., Сеферян К.Г., Гришечкин М.С. Грамотная постановка искусственных зубов с учетом анализа гипсовых моделей беззубых челюстей. // Клиническая стоматология. 2014. № 3 (71). С. 50-52.

5. Демидович Б.П. Численные методы анализа. Приближение функций, дифференциальные и интегральные уравнения. учебное пособие / Б.П. Демидович, И.А. Марон, Э.3. Шувалова; под ред. Б.П. Демидовича. Санкт-Петербург [и др.], 2008. Сер. Классическая учебная литература по математике (Изд. 4-е, стер.)368С.

6. Ермак Е.Ю., Способ создания окклюзионных контактов зубных рядов. Парилов В.В., Ознева Л.М., Индюков В.В. Российский стоматологический журнал. 2010. № 6. С. 10-11.

7. Жолудев С.Е. Особенности протезирования полными съемными протезами и адаптации к ним у лиц пожилого и старческого возраста. // Уральский медицинский журнал. 2012. № 8 (100). С. 31-35.

8. Загорский В.А. Протезирование при полной адентии / В.А. Загорский-М.: Медицина-2008.-218с.

9. Зинякин Р.С. Нечеткое математическое описание физических моделей беззубых челюстей человека / Р.С. Зинякин// В мире научных открытий. —2010. № $6-1 .-$. 75 .

10. Литман В. Теорема Пифагора /В. Литман. М.: Гос. Издат.,1960-116 с.

11. Лола Д.В. Математическое моделирование зубных рядов в полных съёмных протезах./ Д.В. Лола, А.С. Назаров// Вестник СКФУ.-2015.№ 2(47).-с 48-55.

12. Паршин Ю.В. Особенности протезирования нижней беззубой челюсти при сложной клинической картине./ Ю.В. Паршин // Учёные записи СпбГму имени академика И.П. Павлова.-2015.-Т.12,№ 3.-С.11-13.

13. Рединов И.С., Метелица С.И., Страх 0.0. Повышение эффективности повторного лечения пациентов при полном отсутствии зубов на нижней челюсти. // Фундаментальные исследования. 2014. № 10-2. С. 356-359. 
14. Саввиди К.Г. Методика повторного протезирования на беззубой нижней челюсти у пациентов пожилого и старческого возраста с неблагоприятными клиническими условиями протезного ложа. // Институт стоматологии. 2008. № 4 (41). С. 34-35.

15. Тлустенко В.С. Ортопедическое лечение больных при выраженном прогеническом соотношении беззубых челюстей полными съемными протезами. // Аспирантский вестник Поволжья. 2008. № 3-4. С. 170-174.

16. Трунин Д.А., Садыков М.И., Шумский А.В., Нестеров А.М. Ортопедическое лечение пациентов с истонченной, малоподатливой слизистой оболочкой протезного ложа при полном отсутствии зубов. // Институт стоматологии. 2016. № 4 (73). С. 90-91.

17. Трунин Д.А., Садыков М.И., Нестеров А.М., Постников М.А., Сагиров М.Р., Чистякова М.С. Особенности ортопедического лечения больных с полным отсутствием зубов на нижней челюсти с неблагоприятными клиническими условиями. // Медицинский вестник Северного Кавказа. 2017. Т. 12. № 4. C. 421-424.

18. Чижов Ю.В. Полное отсутствие зубов на верхней и нижней челюсти. Возможные сочетания и распространённость у лиц пожилого и старческого возраста./ Ю.В. Чижов, Т.В. Казанцева, Г.И. Николаева // Клиническая Геронтология .-2008.-№ 9.-С.70.

19. Baslas V. Atechnigve for using short tezm soft liners as complete dentures dinal impression material/ V. Baslas, S.V. Singh, H. Aggarwal,//Y. Oral Biol Craniofas. Res.-2014.-V.4(3). -p.204-207

20. Hytsky А. Полные съёмные протезы становятся цифровыми / А. Нytsky // Новое в стоматологии-2016. -№ 7. -С.72-77.

( ) Аксёнов Даниил Игоревич ( p.ghost@rambler.ru ), Майборода Юрий Николаевич, Аксёнов Игорь Николаевич.

Журнал «Современная наука: актуальные проблемы теории и практики»

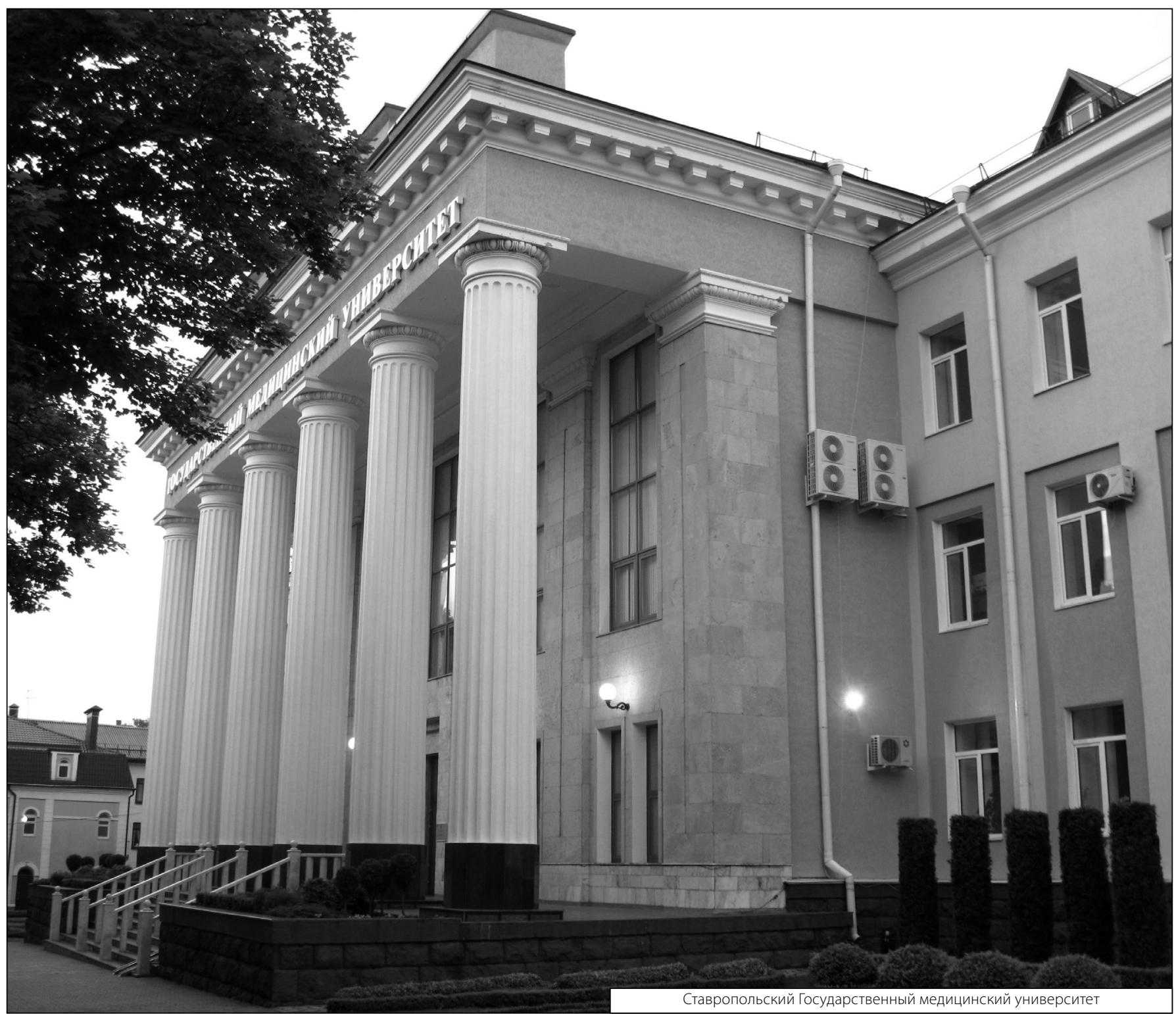

